\title{
COMENTARIOS A LA JURISPRUDENCIA 2a./J. 164/2019 TOPE DE LOS 10 SALARIOS MÍNIMOS A LAS PENSIONES*
}

\author{
COMMENTS TO JURISPRUDENCE 2a./J. 164/2019 MAXIMUM \\ OF THE 10 MINIMUM WAGES FOR PENSIONS
}

COMMENTAIRES SUR LA JURISPRUDENCE 2a./J. 164/2019 RELATIVE AU PLAFOND DES PENSIONS ÉQUIVALANT À 10 FOIS LE SALAIRE MINIMUM

\section{María Ascensión MORALES RAMÍREZ*}

RESUMEN: El 24 de enero de 2020 se publicó, en el Semanario Judicial de la Federación, la tesis jurisprudencial 2a./J. 164/2019, ${ }^{1}$ la cual reitera el tope de 10 veces el salario mínimo para el pago de las pensiones por invalidez, cesantía en edad avanzada y vejez a la generación en transición que opte por el sistema de la Ley del Seguro Social vigente hasta el 30 de junio de 1973, criterio que fue determinado en la jurisprudencia 2a./J.85/2010.

México, en la reforma a la Ley del Seguro Social, a diferencia de los otros países latinoamericanos que introdujeron la capitalización individual en el sistema de pensiones, ${ }^{2}$ no reguló de manera clara y suficiente la situación de la generación en transición, en especial lo relativo al tiempo anterior al ejercicio de la opción de retiro. De esta forma, se negó a los asegurados, al momento de la reforma, la libertad para escoger entre el antiguo y el nuevo sistema. En la exposición de motivos de la ley vigente de 1997 se adujo que "todos los trabajadores en activo empezarían a cotizar bajo el nuevo sistema de pensiones". ${ }^{3}$

* Recibido el 16 de febrero de 2020 y aceptado para su publicación el 25 de marzo de 2020.

** Profesora de Derecho del trabajo y de la seguridad social en la Facultad de Derecho de la UNAM.

1 Dicha tesis corresponde a la ejecutoria pronunciada en sesión del 23 de octubre de 2019, en el expediente relativo a la contradicción de tesis 327/2019.

2 Chile, Perú, Argentina, Colombia, Uruguay, El Salvador, Costa Rica y República Dominicana.

3 IMSS, Ley del Seguro Social, 1996, p. 28. 
15 años después de haberse expedido la Ley del Seguro Social actual (21 de diciembre de 1995), vigente a partir del 1o. de julio de 1997 y evadiendo lo plasmado en la exposición de motivos de esta ley, a través de la jurisprudencia se han ido estableciendo las reglas a las que estará sujeta la generación en transición ante la omisión legislativa, al no preverse un ordenamiento específico al símil de lo que posteriormente resultó ser el Reglamento para el otorgamiento de pensiones de los trabajadores sujetos al régimen del artículo décimo transitorio de la Ley del Instituto de Seguridad y Servicios Sociales de los Trabajadores del Estado (LISSSTE), el cual fue producto de 223,810 amparos, ${ }^{4}$ que incluían al 90\% de los asegurados.

Ante esta imprecisión e incertidumbre jurídica, en la práctica, las acciones del Instituto Mexicano del Seguro Social (IMSS) en sus cálculos, negativas, modificación, rectificación y pago correcto del monto o incremento de la pensión, han obligado a los asegurados a acudir a las instancias jurisdiccionales.

Así, mediante cuatro jurisprudencias de la Segunda Sala de la Suprema Corte de Justicia de la Nación (SCJN), ${ }^{5}$ a saber: 2a./J.85/2010; 2a./J. ${ }^{6}$ 114/2012; 2a./J.8/2016, , y 2a./164/2019,", se ha configurado el "régimen transitorio" de los afiliados al IMSS al amparo de la Ley del Seguro Social vigente hasta el 30 de junio de 1997 y, por ende, la determinación del tope aplicable al cálculo y pago de las pensiones.

Palabras clave: jurisprudencia, tope, salario mínimo, pensiones, seguridad social.

4 En 2007 existían dos millones 800 mil asegurados, según se expresó en la exposición de motivos de la ley.

5 Tres, resultado de contradicción de tesis: 2a./J.85/2010; 2a./J.8/2016, y 2a./164/2019.

6 Seguro Social. El salario de las últimas 250 semanas de cotización, base para cuantificar las pensiones por invalidez, vejez y cesantía en edad avanzada tiene como límite superior el equivalente a 10 veces el salario mínimo general vigente en el Distrito Federal, acorde con el segundo párrafo del artículo 33 de la ley relativa, vigente hasta el 30 de junio de 1997.

7 Seguro Social. Régimen transitorio del sistema de pensiones entre las leyes del Seguro Social, derogada y vigente. Sus diferencias.

8 Pensión por cesantía en edad avanzada. Al resolver la junta sobre el ajuste en la cuantificación de su pago, se debe atender el límite superior previsto en el párrafo segundo del artículo 33 de la Ley del Seguro Social, vigente hasta el 30 de junio de 1997, sin que represente obstáculo alguno que el demandado no se hubiere excepcionado en ese sentido.

9 Régimen transitorio del sistema de pensiones entre las leyes del Seguro Social, derogada de 1973 y vigente. El límite superior que se debe aplicar al salario promedio de las 250 semanas de cotización que sirve de base para cuantificar las pensiones de los asegurados del régimen transitorio que optaron por el esquema pensionario de la derogada Ley de 1973. 
ABSTRACT: On January 24, 2020, the jurisprudential thesis 2/J 164/2019, was published in the Judicial Weekly of the Federation. which reiterates the maximum of 10 times the minimum wage for the payment of pensions for disability, unemployment in old age and old age to the generation in transition who chooses for the Social Security Law system in force until June 30, 1973, criterion that was determined in jurisprudence 2a./J.85/2010.

In the reform of the Social Security Law, Mexico, unlike the other Latin American countries that introduced individual capitalization in the pension system, did not clearly and sufficiently regulate the situation of the generation in transition, especially the relative at the time prior to exercising the retirement option. Thus, at the time of the reform, policyholders were denied the freedom to choose between the old and the new system. In the explanatory memorandum of the current law of 1997, it was argued that "all active workers would start contributing under the new pension system".

15 years after the issuance of the current Social Security Law (December 21, 1995), effective as of July 1st, 1997 and evading what is stated in the explanatory memorandum of this law, through jurisprudence it has been establishing the rules to which the generation in transition will be subject to due to legislative omission, as there is no specific order similar to what later turned out to be the Regulations for the granting of pensions to workers subject to the regime of the Tenth Transitory Article of the Law of the Institute of Security and Social Services of State workers (LISSSTE), which was the product of 223,810 amparos 4 that included the $90 \%$ of the insured.

Under this imprecision and legal uncertainty, in practice, the actions of the Mexican Institute of Social Security (IMSS) in the calculations, refusals, modification, rectification and correct payment of the amount or increase of the pension, have forced the insured to go to the jurisdictional courts.

Thus, through four jurisprudences of the Second Chamber of the Supreme Court of Justice of the Nation (SCJN), 5 namely: 2a./J.85/2010; 2a./J. 114/2012; 2a./J.8/2016; and 2a./164/2019, the "transitional regime" of IMSS affiliates has been set up under the Social Security Law in force until June 30, 1997 and, therefore, the determination of the maximum applicable to the calculation and payment of pensions.

Keywords: jurisprudence, stop, minimum salary, pensions, social security. 
RÉSUMÉ: Le 24 janvier 2020, le Semanario Judicial de la Federación (hebdomadaire du Mexique qui permet de faire connaître les différentes jurisprudences adoptées) a publié la thèse jurisprudentielle 2a./J. 164/2019 qui confirme de nouveau que le montant des pensions d'invalidité et des allocations vieillesse ne peut excéder 10 fois le salaire minimum pour les générations de salariés qui choisiront le régime de retraite défini par la Loi sur la Sécurité Sociale (Ley del Seguro Social) en vigueur jusqu'au 30 juin 1973, critère précédemment établi par la jurisprudence 2a./J.85/2010.

Contrairement aux autres pays latinoaméricains qui ont opté pour la capitalisation individuelle dans le système des pensions, le Mexique n'avait pas déterminé de règles claires et transparentes en ce qui concerne la génération de salariés qui feront prochainement valoir leurs droits à la retraite en omettant de se prononcer sur leurs dernières années de travail. En conséquence, les assurés sociaux, au moment de la réforme, n'ont pas eu la liberté de choisir entre le nouveau système de retraite et l'ancien. Rappelons que dans le préambule de la loi de 1997 en vigueur, il est avancé que "tous les travailleurs actifs commenceraient à cotiser dans le cadre du nouveau système des retraites".

15 ans après avoir déposé cette Loi sur la sécurité sociale (21 décembre 1995) toujours actuelle et entrée en vigueur le 1er juillet 1997, la jurisprudence, ignorant ce qui figure dans le préambule, adopta de nouvelles règles que la génération de salariés qui feraient prochainement valoir leurs droits à la retraite devaient respecter parce que la Loi ne s'était pas prononcée sur leur statut. Telle fut la situation tant que n’apparaissait pas une réglementation spécifique et similaire au Règlement pour l'attribution des pensions de retraite des travailleurs relevant du régime défini dans le 10ème article transitoire de la Loi de l'Institut de la Sécurité Sociale et des Services Sociaux des Fonctionnaires Publics (Ley del Instituto de Seguridad y Servicios Sociales de los trabajadores del Estado, LISSSTE), règlement qui fut le produit de 223810 demandes de protection (amparos) pour pouvoir inclure $90 \%$ des assurés.

Face à une telle imprécision et à une telle incertitude juridique, l'Institut Mexicain de la Sécurité Sociale (Instituto Mexicano del Seguro Social, IMSS) qui, dans la pratique, devait procéder à des calculs, des prélèvements, des modifications, des rectifications et aux versements corrects des montants ou des majorations de pension, a provoqué de nombreuses réclamations de la part des assurés qui ont eu recours à divers organes juridictionnels.

C'est la raison pour laquelle quatre jurisprudences ont vu le jour dans la deuxième salle de la Cour suprême de la Justice du Mexique (Suprema Corte de Justicia de la Nación, SCJN), les jurisprudences 2a./J.85/2010; 
2a./J. 114/2012; 2a./J.8/2016, y 2a./164/2019, afin de définir un "régime transitoire" pour les affiliés à l'IMSS dans le cadre de la Loi sur la sécurité sociale en vigueur jusqu'au 30 juin 1997 et pour fixer le plafond applicable au calcul et au versement des pens.

Mots clés: jurisprudence, arrêtez, salaire minimum, pensions, sécurité sociale.

SuMARIO: I. La jurisprudencia 2a.164/2019. II. Configuración de la regulación de la generación en transición. III. El IMSS frente a la jurisprudencia. IV. Reflexiones finales.

\section{LA JURISPRUDENCIA 2A.164/2019}

\section{Posiciones interpretativas}

L

a jurisprudencia en cuestión es el resultado de una contradicción de criterios entre los sustentados por el Tribunal Colegiado en Materias Penal y de Trabajo del Octavo Circuito y el Decimosexto Tribunal Colegiado en Materia de Trabajo del Primer Circuito ${ }^{10}$ vs. el Segundo Tribunal Colegiado en Materia de Trabajo del Tercer Circuito. ${ }^{11}$

El primer criterio consideró

...que para cuantificar el monto de la pensión de vejez tratándose de asegurados que se ubican en el régimen transitorio y que se acogieron al esquema de pensiones establecido en la derogada Ley del Seguro Social (1973), resulta aplicable el tope máximo de 10 veces el salario mínimo previsto en el artículo 33, segundo párrafo, del citado cuerpo normativo, así como la jurisprudencia 2a./J. 85/2010, de la Segunda Sala de rubro: "SEguro SoCIAL. EL SALARIO PROMEDIO DE LAS ÚLTIMAS 250 SEMANAS DE COTIZACIÓN, BASE PARA CUANTIFICAR LAS PENSIONES POR INVALIDEZ, VEJEZ Y CESANTÍA EN

10 En el primer tribunal colegiado de circuito, el caso era el cambio de una pensión de cesantía en edad avanzada por una de vejez. En el segundo, la reducción de la pensión de vejez que había disfrutado el quejoso, y dos años después, se le redujo para cuantificarla a razón de 10 salarios mínimos.

11 El caso se trató de una rectificación y pago correcto de la pensión de cesantía en edad avanzada y vejez. 
EDAD AVANZADA, TIENE COMO LÍMITE SUPERIOR EL EQUIVALENTE A 10 VECES EL SALARIO MÍNIMO GENERAL VIGENTE EN EL DISTRITO FEDERAL, ACORDE CON EL SEGUNDO PÁRRAFO DEL ARTÍCULO 33 DE LA LEY RELATIVA, VIGENTE HASTA EL 30 DE JUNIO DE 1997"; y por consiguiente no se actualiza el supuesto del artículo vigésimo quinto transitorio, de la Ley del Seguro Social de 1997, el que sólo rige para los asegurados que opten por pensionarse conforme al esquema establecido en la Ley del Seguro Social citada en último término.

El segundo criterio estimó que para cuantificar el monto de la pensión de vejez, tratándose de asegurados que se ubican en el régimen transitorio y que se acogieron al esquema de pensiones establecido en la derogada Ley del Seguro Social (1973), no resultaba aplicable el tope máximo de 10 veces el salario mínimo previsto en el artículo 33, segundo párrafo, del citado cuerpo normativo, ni la jurisprudencia 2a./J. 85/2010, y, por consiguiente, sí se actualizaba el supuesto del artículo vigésimo quinto transitorio de la Ley del Seguro Social de 1997.

\section{Criterio imperante}

La Segunda Sala de la SCJN arribó a la conclusión de que para cuantificar el monto de la pensión de vejez, tratándose de asegurados que se ubican en el régimen transitorio y que se acogieron al esquema de pensiones establecido en la derogada Ley del Seguro Social (1973), sí resulta aplicable el tope máximo de 10 veces el salario mínimo previsto en el artículo 33, segundo párrafo, del citado cuerpo normativo, así como la jurisprudencia 2a./J. 85/2010, de esta Segunda Sala, de rubro:

\footnotetext{
SEguro Social. El SAlario Promedio de las Últimas 250 SEMANAS DE COTIZACIÓN, BASE PARA CUANTIFICAR LAS PENSIONES POR INVALIDEZ, VEJEZ Y CESANTÍA EN EDAD AVANZADA, TIENE COMO LÍMITE SUPERIOR EL EQUIVALENTE A 10 VECES EL SALARIO MÍNIMO GENERAL VIGENTE EN EL DiSTRITO FEDERAL, ACORDE CON EL SEGUNDO PÁRRAFO DEL ARTÍCUlO 33 DE LA LEY RELATIVA, VIGENTE HASTA EL 30 DE JUNIO DE 1997.
}

Puesto que el precepto aludido está contenido en la ley que regula el régimen pensionario que se eligió, motivo por el que si se escoge el esquema 
previsto en una legislación es indudable que las disposiciones de ésta son las que deben regir lo relacionado con la pensión; además de que la citada jurisprudencia se basa en un precepto que está inmerso en la normativa con base en la cual se regula la pensión, de ahí que también sea aplicable.

Así, prevaleció con carácter de jurisprudencia este criterio, dando lugar a la tesis $164 / 2019$.

\section{CONFIGURACIÓN DE LA REGULACIÓN \\ DE LA GENERACIÓN EN TRANSICIÓN}

\section{Interpretación de la SCJN}

La Segunda Sala, con las jurisprudencias 85/2010, de julio de 2010, y 114/2012, de octubre de 2012, configuró la regulación de los asegurados hasta el 30 de junio de 1997, respecto al límite de la cuantificación y pago de sus pensiones, conforme a los artículos siguientes: ${ }^{12}$

a) Artículos 33, segundo párrafo, y 167 de la Ley de 1973.

La Segunda Sala señala que, pese a la derogación de la Ley del Seguro Social de 1973, ésta no deja de tener efectos jurídicos para los retirados durante su vigencia y para aquellos asegurados que al pensionarse eligieron el esquema establecido en dicha ley. $\mathrm{Al}$ respecto precisa que:

- El artículo 33 establece dos topes salariales: ${ }^{13}$

- En su primer párrafo, un tope equivalente a 25 veces el salario mínimo general vigente para el entonces Distrito Federal para los ramos de retiro, enfermedad general y maternidad.

- En su segundo párrafo, un tope equivalente a 10 veces el salario mínimo general vigente para el entonces Distrito Federal para los seguros de invalidez, vejez y cesantía en edad avanzada.

- El artículo 167 establece que el salario diario que servirá de base para determinar la cuantía básica de las pensiones de invalidez, vejez y cesantía en edad avanzada será el promedio de las últimas 250 semanas de cotización; pero en caso de que no tuviera el asegurado ese promedio, se considerarían las que tuviera acreditadas,

12 Tal criterio fue reiterado en las dos jurisprudencias restantes.

13 El contenido de este artículo corresponde a la reforma del 20 de julio de 1993. 
siempre que sean suficientes para el otorgamiento de la pensión correspondiente. ${ }^{14}$

b) Artículos transitorios de la Ley de 1997. Tercero, cuarto, undécimo, duodécimo y décimo tercero, los cuales tienen en común la opción para optar entre el sistema de pensiones de 1973 o el de 1997.

Con base en tales disposiciones, la Segunda Sala estableció que el pago de las pensiones debe ser hasta 10 veces el salario mínimo, a pesar de que los artículos 33, segundo párrafo, y 167 de la Ley de 1973 regulaban el inicio (la inscripción y salario base de cotización) y permanencia de la vida laboral. Por su parte, los transitorios previstos en la Ley de 1997 regulan el final de la vida laboral.

\section{Sistema híbrido}

La Segunda Sala no se remite al contexto histórico de la reforma al sistema de pensiones y al debate nacional e internacional sobre la situación en que se dejó a la generación en transición al no establecerse las disposiciones específicas que aplicarían en tanto no se llegara al final de la vida laboral. ${ }^{15}$ Así, al entrar en vigor la Ley de 1997 en forma automática, los asegurados hasta el 30 de junio de 1997 fueron objeto de regulación de la nueva ley y de sus parámetros:

a) Tuvieron que abrir una cuenta individual en donde se depositarían sus cotizaciones, las de sus patrones y las del gobierno federal.

b) El seguro al cual cotizaban invalidez, vejez, cesantía en edad avanzada y muerte (IVCM) se dividió en dos seguros: de invalidez y vida (I y V) y de retiro, cesantía en edad avanzada y vejez (RCV).

c) El financiamiento de sus pensiones cambió: a partir de 1997 sus cotizaciones experimentaron aumentos graduales, de conformidad con el artículo vigésimo quinto transitorio, el cual estableció que en lo relativo al seguro de invalidez y vida, así como en los ramos de cesantía en edad avanzada y vejez, a partir de la entrada en vigor de la ley, el límite del salario base de cotización en veces salario mínimo sería de

14 El texto del artículo 167 señala: "Para los efectos de determinar la cuantía básica anual de la pensión y sus incrementos; se considera como salario diario el promedio correspondiente a las últimas doscientas cincuenta semanas de cotización".

15 Bonilla García, Alejandro y Conte-Grand, Alfredo (comps.), Pensiones en América Latina. Dos décadas de reforma, OIT, 1998. 
15 veces el salario mínimo general vigente en el Distrito Federal, el que se aumentaría un salario mínimo por cada año subsecuente hasta llegar a 25 en el año 2007.

\begin{tabular}{|c|c|}
\hline Año & $\begin{array}{c}\text { Veces el salario minimo } \\
\text { del Distrito Federal }\end{array}$ \\
\hline 1997 & 15 \\
\hline 1998 & 16 \\
\hline 1999 & 17 \\
\hline 2000 & 18 \\
\hline 2001 & 19 \\
\hline 2002 & 20 \\
\hline 2003 & 21 \\
\hline 2004 & 22 \\
\hline 2005 & 23 \\
\hline 2006 & 24 \\
\hline 2007 & 25 \\
\hline
\end{tabular}

Lo anterior porque el artículo 28 de la Ley de 1997 vigente estableció el límite superior en 25 salarios mínimos para efectos del cálculo del salario base de cotización, y el mismo entraría en vigor hasta el 2007 para las pensiones de invalidez y vida, cesantía en edad avanzada y vejez.

Artículo 28. Los asegurados se inscribirán con el salario base de cotización que perciban a momento de su afiliación, estableciéndose como límite superior el equivalente a veinticinco veces el salario mínimo general que rija en el Distrito Federal y como límite inferior el salario mínimo general del área geográfico respectiva.

d) No se reguló un bono de reconocimiento de los periodos aportados al antiguo sistema, dejando una incertidumbre jurídica. La reforma estableció para el asegurado, al final de la vida laboral, la opción de pensionarse con el esquema de 1973 o el de 1997, previo cálculo de ambas opciones (artículos transitorios tercero y décimo primero).

En su momento se argumentó que el tratamiento legal de los derechos adquiridos hacía difícil asignarles un valor a los beneficios generados bajo el antiguo sistema hasta el momento de la reforma, pero al estar reconocidos 
en el nuevo sistema no se daba margen a una interpretación legal sobre el valor de éstos. ${ }^{16}$

Lo expuesto evidencia que los parámetros para la generación en transición cambiaron y son objeto de regulación de un sistema híbrido:

- Aplicación de las normas de la Ley de 1997 durante la vida laboral, entre ellos el límite superior de 25 salarios mínimos.

- Aplicación del sistema de pensiones de la Ley de 1973 al final de la vida laboral, únicamente para efectos de ejercer su derecho de opción en lugar de la pensión por cuentas individuales.

A pesar de ello, la Segunda Sala de la SCJN, en la jurisprudencia 164/19, determina que si el trabajador opta por pensionarse por el sistema regido por la Ley de 1973, aunque baya seguido cotizando bajo el régimen de la nueva Ley de 1997 (para el que haya cotizado arriba de 10 y hasta 25 salarios mínimos), recibirá su pensión en términos de la Ley de 1973, dado que esas pensiones deben otorgarse bajo los parámetros fijados en las disposiciones de dicho cuerpo legal (tope de 10 salarios mínimos previsto en el artículo 33, segundo párrafo) y no con el artículo 28 y vigésimo quinto transitorio (tope de 25 salarios), en virtud de que el financiamiento del régimen de pensión anterior y el nuevo son distintos, motivo por el cual a cada uno se le debe aplicar la normativa correspondiente a su esquema pensionario.

Este argumento es contradictorio, porque los pensionados financiaron sus pensiones con las disposiciones aplicables, en su momento, por ambas leyes y, en forma específica, arriba de 10 salarios mínimos desde 1997.

Dicha Sala, en la jurisprudencia 2a./J 85/2010, señaló que en la reforma del 20 de julio de 1993 la intención del legislador fue establecer, en el segundo párrafo del artículo 33, el límite de 10 veces el salario mínimo como salario base de cotización a los seguros de invalidez, cesantía en edad avanzada y vejez, y por ello el salario base para cuantificar las pensiones no podría rebasar ese tope.

Sin embargo, también hace referencia a que en la exposición de motivos de la Ley de 1973 se aludió a "que tanto para el pago de las cuotas, como para el reconocimiento de derechos y el otorgamiento de las prestaciones en dinero, el salario es la base de cotización”. Empero, evade considerar tal salario, como es el hecho de que a partir de 1997 la generación en transición

16 IMSS, cit. 
cotizó con un salario superior a los 10 salarios mínimos y teniendo como límite las 25 veces tal salario, porque desde la exposición de motivos de dicha ley se adujo que empezarían a cotizar en el nuevo sistema, razón por la que les aplicaron el artículo vigésimo quinto (incremento gradual de las cotizaciones).

La Segunda Sala concluye puntualizando que el ámbito de aplicación de la jurisprudencia 2a./J 85/2010, es decir, el tope de 10 salarios mínimos para el cálculo y pago de las pensiones:

- Aplica a los asegurados del régimen de mil novecientos setenta y tres que hayan cotizado conforme a las reglas del régimen de mil novecientos noventa y siete y hayan optado por el régimen de 1973 (los que hayan cotizado arriba de 10 y hasta 25 salarios mínimos).

- No aplica a los asegurados del régimen de mil novecientos setenta y tres que hayan cotizado conforme a las reglas del régimen de mil novecientos noventa y siete y hayan optado por este último régimen.

Pese al reconocimiento de que la generación en transición cotizó conforme a la Ley de 1997, esto es, para efectos recaudatorios conforme al artículo 28 y vigésimo quinto transitorio, la Segunda Sala de la SCJN determina que para el pago de pensiones no son aplicables tales disposiciones. Asimismo, señala que el artículo vigésimo quinto transitorio de la Ley de 1997 aplica para los asegurados que opten por pensionarse conforme al esquema de pensiones establecido en dicha ley, en oposición a lo señalado en la exposición de motivos de esta ley.

Empero, en ninguna de sus resoluciones dicha Sala se ha pronunciado respecto del excedente entre los más de 10 y los 25 salarios mínimos que cotizaron los asegurados afiliados antes del 1o. de julio de 1997, lo cual pudiera llegar a ser materia de demanda laboral o administrativa.

Por lo que hace al criterio jurisprudencial de que el tope de los 10 salarios mínimos "no aplicará a los asegurados del régimen de 1973 y que hayan cotizado conforme a las reglas del régimen de 1997 y hayan optado por este último régimen" resulta irrelevante, porque la pensión bajo el sistema de cuentas individuales dependerá del ahorro acumulado; entonces, no se necesita un límite para el cálculo de su pensión.

No obstante, pesará sobre los pensionados que elijan el sistema de 1997 el que no se les haya otorgado un "bono de reconocimiento" de los periodos cotizados en el anterior sistema, pues el ahorro acumulado en las cuentas 
individuales es muy poco, como ya lo ha evidenciado la Comisión Nacional del Sistema de Ahorro para el Retiro (Consar) respecto de la primera generación de ese sistema. ${ }^{17}$ Quizá sea esta la razón por la cual los pensionados de la generación en transición lo han hecho bajo el régimen de la Ley de 1973.

\section{EL IMSS FRENTE A LA JURISPRUDENCIA}

Desde el inicio de estas jurisprudencias en 2010, el titular del IMSS en turno ha emitido diversos comunicados de prensa, en los que, en un principio, aducía que se analizarían las implicaciones del criterio jurisprudencial para los futuros pensionados y, con base en ello, actuaría conforme a las disposiciones de la Ley del Seguro Social. Posteriormente, con motivo de la jurisprudencia 8/2016, se precisaron tres puntos: a) la jurisprudencia no alteraría el mecanismo de cálculo para el pago de las pensiones, de tal suerte que se garantizaban los derechos de los pensionados; b) los ya pensionados continuarían recibiendo de manera puntual su pensión, y c) se continuaría aplicando el tope de los 25 salarios mínimos en el pago de las pensiones.

Así, desde ese entonces el titular en turno ha expresado que la jurisprudencia no obliga o altera el cálculo de las pensiones y, por tanto, no aplicará éstas respecto al tope de 10 salarios mínimos para cuantificar y pagar las pensiones al amparo de la Ley del Seguro Social vigente al 30 de junio de 1997. ${ }^{18}$ Sirven de ejemplo las declaraciones siguientes:

...el IMSS pagará las pensiones de todos sus afiliados, sin importar si se jubilan (sic) por el régimen de la Ley de 1973, con un tope de 25 veces el salario mínimo vigente en el Distrito Federal. ${ }^{19}$

Se va a respetar el esquema de cotización de todos los trabajadores que están en este esquema de transición a la hora de cotizar arriba de 10 salarios mínimos, es decir, se les va a seguir calculando en 25, por una simple razón: creemos que es el trabajo de toda su vida que cotizaron con 25 salarios, pues sería injusto reducirlo a 10, pero la sentencia, esta controversia de tesis no es aplicable al Seguro Social. ${ }^{20}$

17 En 2021, de 750 mil asegurados, sólo el 1\% (750) alcanzará una pensión. El 99\% (74,250) tendrán que seguir laborando o retirar sus ahorros en una sola exhibición.

18 IMSS, Boletín de Prensa, 30 de junio de 2010.

19 Arriola, Mikel, disponible en: https/ / www.excelsior.com.mx/ nacional/2016/05/17/1093085.

20 Robledo, Zoé, conferencia de prensa del 4 de enero de 2020. 
Entonces, con motivo de la jurisprudencia por contradicción de tesis 2a./164/2019, del 24 de enero de 2020, el Consejo Técnico de IMSS, en sesión extraordinaria celebrada el 5 de febrero de 2020,21 ratificó el criterio aplicado por el instituto para considerar los 25 salarios mínimos como límite superior para el pago de las pensiones al amparo de la Ley de 1973.

Sin embargo, no hay que olvidar que ante la impugnación en la vía jurisdiccional de los errores del instituto en el cálculo de las pensiones, o sus negativas a otorgarlas o a corregir y subsanar las diferencias, los representantes legales del IMSS son los que han solicitado la aplicación del tope de 10 salarios mínimos.

\section{REFLEXIONES FINALES}

La medida adoptada por el Consejo Técnico de IMSS es insuficiente porque no se resuelve la incertidumbre jurídica hacia adelante; esto es, para los trabajadores en activo (generación en transición) que en su momento decidan elegir la Ley de 1973, ni tampoco los errores de cálculo en que incurra el instituto.

Es necesario que se expida y se publique, en el Diario Oficial de la Federación, un reglamento que precise las condiciones de cotizaciones, otorgamiento e incremento de los beneficios a que tiene derecho la generación en transición de 1973, pues ante una acción u omisión del IMSS en materia de pensiones en perjuicio de los pensionados, se da como un supuesto que ante un litigio en contra del instituto las instancias jurisdiccionales aplicarán la jurisprudencia del tope de los 10 salarios mínimos, a pesar de que se haya cotizado con los parámetro de la Ley de 1997.

Adicionalmente, hasta ahora no se ha planteado el hecho de que los incrementos anuales a las pensiones de la generación en transición se han realizado conforme al aumento de la unidad de medida y actualización (UMA) y no con base en los salarios mínimos, como lo establece la Ley de 1973, situación que afecta considerablemente a las pensiones más bajas, pues el incremento de la UMA para 2020 fue de $2.83 \%, 22$ mientras que de los salarios mínimos fue de $20 \%$ (14.67\% del monto independiente de recuperación, más el 5\% de aumento).

21 IMSS, Boletín de Prensa, núm. 064/2020, 6 de febrero de 2020.

22 Inegi, comunicado de prensa, núm. 008/20, 9 de enero de 2020. 
Por último, con la decisión del Consejo Técnico de aplicar los 25 salarios mínimos podría entonces entenderse que ¿el IMSS dejará de aplicar la UMA para la cotización, cálculo e incremento de las pensiones?, pues un tema que está en debate al verse afectadas todas las pensiones, y en especial las más bajas, es que el porcentaje de incremento anual que les aplican es con base en dicha medida y no con el salario mínimo. 